$\overline{\mathrm{AEET}}$

ASOCIACIÓN ESPAÑOLA

DE ECOLOGÍA TERRESTRE
Ecosistemas 21(3):31-40 [Septiembre-Diciembre 2012] Doi.: 10.7818/ECOS.2012.21-3.05

Artículo publicado en Open Access bajo los términos de Creative Commons attribution Non Comercial License.

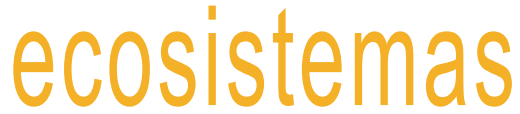

REVISTA CIENTÍFICA DE ECOLOGÍA Y MEDIO AMBIENTE

\title{
Vulnerabilidad frente a la sequía de repoblaciones de dos especies de pinos en su límite meridional en Europa
}

\author{
R. Sánchez-Salguero',2,*, R.M. Navarro-Cerrillo², J.J. Camarero³, A. Fernández-Cancio ${ }^{1}$, T.W. Swetnam ${ }^{4}$, M.A. Zavala ${ }^{5}$ \\ (1) Centro de Investigación Forestal (CIFOR), INIA. Carretera de la Coruña km. 7, 28040, Madrid, España. \\ (2) Depto. Ingeniería Forestal, ETS Ing. Agrónomos y de Montes. Univ. Córdoba. Campus de Rabanales, Crta. IV, km. 396, 14071 Córdoba. España. \\ (3) ARAID-Instituto Pirenaico de Ecología, CSIC. Avda. Montañana 1005, 50192 Zaragoza. España. \\ (4) Laboratory of Tree-Ring Research, 105 West Stadium, University of Arizona, Tucson, AZ 85721, United States. \\ (5) Departamento de Ecología, Facultad de Ciencias, Universidad de Alcalá. 28871. Alcalá de Henares, Madrid, España.
}

* Autor de correspondencia: R. Sánchez-Salguero [rsanchez@uco.es]

> Recibido el 2 de octubre de 2012, aceptado el 30 de noviembre de 2012

Sánchez-Salguero, R., Navarro-Cerrillo, R.M., Camarero, J.J., Fernández-Cancio, A., Swetnam, T.W., Zavala, M.A. (2012). Vulnerabilidad frente a la sequía de repoblaciones de dos especies de pinos en su límite meridional en Europa. Ecosistemas 21(3):31-40. Doi.: 10.7818/ECOS.2012.21-3.05.

El actual cambio climático conlleva un aumento de eventos climáticos extremos como las sequías que pueden desencadenar procesos de decaimiento forestal, particularmente en sitios xéricos como los situados en el límite sur de distribución de especies de árboles. La vulnerabilidad de las repoblaciones de pinos realizadas en la alta montaña Mediterránea frente a las sequías está condicionada por esta variabilidad climática que afecta a su crecimiento, vigor y persistencia a largo plazo. En este trabajo se analiza el impacto de diversas sequías recientes (1994, 1995, 1999 y 2005) sobre el crecimiento radial y el vigor de dos especies de pino (Pinus sylvestris, Pinus nigra) en zonas semiáridas del sudeste de la Península lbérica (Sierra de los Filabres), cerca de su límite meridional de distribución. Se usaron datos dendrocronológicos y de defoliación para cuantificar, por medio de modelos logísticos y modelos lineales mixtos, las respuestas del crecimiento y vigor de los árboles, a escalas de individuo y especie, respecto a la estructura del rodal, las condiciones del sitio (intensidad de competencia) y la sequía. En el sudeste ibérico un aumento de temperatura y una disminución en las precipitaciones de primavera han dado lugar a unas condiciones más secas durante finales del siglo XX. Los niveles de defoliación y las reducciones en el crecimiento fueron mayores en la especie más vulnerable a la embolia del xilema inducida por déficit hídrico $(P$. sylvestris) que en la más resistente ( $P$. nigra). El crecimiento radial fue favorecido por condiciones húmedas a finales de primavera (mayo) y comienzos de verano (junio) en ambas especies. El crecimiento de los árboles más defoliados respondió más al déficit hídrico y a la competencia que el de los árboles menos defoliados. Además, el clima y la competencia fueron los dos factores que más afectaron el crecimiento. La fuerte reducción del crecimiento radial junto con la defoliación generalizada fueron los principales componentes de los episodios de decaimiento descritos en las repoblaciones de pino estudiadas. El decaimiento de repoblaciones de pinos en sitios semiáridos del sudeste ibérico, donde experimentan sequías frecuentes y extremas, cuestiona su persistencia futura bajo los escenarios climáticos predichos para este siglo y caracterizados por condiciones más cálidas y áridas.

Palabras clave: Cambio climático, dendroecología, anchura de anillo, defoliación, estrés hídrico, decaimiento.

Sánchez-Salguero, R., Navarro-Cerrillo, R.M., Camarero, J.J., Fernández-Cancio, A., Swetnam, T.W., Zavala, M.A. (2012). Vulnerability to drought of two pine species in plantations at the rear edge of Europe. Ecosistemas 21(3):31-40. Doi.: 10.7818/ECOS.2012.21-3.05.

Climate change causes an increase of extreme weather events such as droughts that can trigger processes of forest decline, especially in droughtprone areas such as the southern limit of distribution of tree species. The vulnerability of Mediterranean mountain plantations to drought is conditioned by this climatic variability, which ultimately affects growth, vigor and long-term persistence. In this paper, we analyze the impact of several recent droughts (1994, 1995, 1999 and 2005) on the radial growth and vigor of two pine species (Pinus sylvestris, Pinus nigra) in a semi-arid mountain at southeastern Iberian Peninsula (Sierra Filabres), near their rear-edge limit. We used dendrochronological and defoliation data to quantify, by means of logistic and linear mixed models, growth and vigor changes at both individual and species scales in response to stand structure, site conditions (competition) and drought. In the lberian southeast, a temperature rise and a decrease in spring precipitation have led to drier conditions during the late twentieth century. Defoliation levels and reductions in growth were higher in the species more vulnerable to drought-induced xylem embolism $(P$. sylvestris) than in the most resistant one ( $P$. nigra). The radial growth was favored by wet conditions in late spring (May) and early summer (June) in both species. The growth of defoliated trees responded more to water deficit and competition than that of less defoliated trees. Climate and competition were the two main factors driving growth. The sharp growth reduction and widespread defoliation were the main components of the decline episodes described in the studied pine plantations. Current decline in pine plantations in the southeastern Iberian Peninsula, where these stands experience severe and frequent droughts, calls into question their persistence in the face of the forecast climatic scenarios for the current century, characterized by warmer and more arid conditions.

Key words: Climatic Change, dendroecology, tree-ring width, defoliation, drought stress, afforestation, decline. 


\section{Introducción}

Los bosques son ecosistemas claves como reguladores a escala global de los ciclos del agua y del carbono, pero también pueden constituir monitores de los efectos del cambio global sobre los ecosistemas terrestres si responden de forma fidedigna a los cambios recientes de clima, de usos del suelo y a las modificaciones de los ciclos biogeoquímicos (Bonan 2008). El cambio climático producirá un aumento de las temperaturas, una mayor frecuencia de sequías y un aumento de la variabilidad climática en muchas regiones del mundo, siendo acentuados sus efectos en la cuenca Mediterránea (IPCC 2007). En la actualidad se prevé que el cambio climático cause una frecuencia cada vez mayor de episodios de decaimiento forestal y mortalidad de los bosques, particularmente en zonas sometidas a estrés hídrico como la cuenca Mediterránea (Allen et al. 2010).

Dicha tendencia a una mayor aridez asociada al aumento de estrés hídrico es previsible que provoque un descenso en la tasa de fijación de carbono de los árboles y una reducción del crecimiento. Esta respuesta fisiológica se ha relacionado con síntomas de decaimiento y un incremento de la mortalidad en diversas especies (Peñuelas et al. 2001), siendo estos efectos más acusados en aquellas poblaciones situadas en el límite sur de su distribución, como es el caso de Pinus sylvestris L. en la Península Ibérica (Martínez-Vilalta et al. 2008, 2012; Vilà-Cabrera et al. 2011; Camarero et al. 2012; Sánchez-Salguero et al. 2012 a,b).

Es previsible que estas poblaciones meridionales sean especialmente susceptibles al creciente estrés climático y se vean afectadas por el aumento de densidad de los bosques debido al abandono de la gestión rural o a la falta de tratamientos selvícolas adecuados en las repoblaciones o masas artificiales (Linares et al. 2009, 2010; Camarero et al. 2011). Pese a su importancia ecológica y económica y su extensión se ha evaluado escasamente el efecto del clima sobre el decaimiento de repoblaciones forestales.

En la Península Ibérica el área ocupada por pino silvestre es de 1377716 ha y aproximadamente 772516 ha (56\%) son áreas repobladas (Pemán y Navarro-Cerrillo, 1997). En cuanto al pino laricio (Pinus nigra Arnold. subsp. salzmannii (Dunald) Franco), la superficie cubierta por esta especie es de 1242388 ha de las cuales 358500 ha (31\%) son repobladas (Pemán y Navarro-Cerrillo 1997). Estas áreas repobladas se presentan como excelentes sistemas experimentales para entender la sensibilidad, vulnerabilidad y capacidad adaptativa al cambio climático de los ecosistemas forestales españoles, ya que se trata de bosques estructural y genéticamente menos variables que los bosques naturales $(\mathrm{He}-$ lama et al. 2008).

Estas masas forestales repobladas pueden encontrarse fuera del límite de distribución natural de la especie lo que podría aumentar su sensibilidad al clima y predisponerlas al decaimiento (Fernández-Cancio et al. 2011). Además, la viabilidad futura de la repoblación puede depender mucho de la variabilidad climática de las décadas iniciales, cuando se produce su establecimiento, y del manejo realizado, o del grado de competencia al que se ven sometidos los árboles durante la primeras etapas de su desarrollo (Serrada et al. 2008).

El actual escenario de cambio climático y escasa intervención silvícola parece ser particularmente relevante para entender la viabilidad futura de muchas repoblaciones de pinos en la cuenca del Mediterráneo. Así, en el año 2001 se detectó un proceso de decaimiento manifestado en forma de amarilleamiento y defoliación intensos y de mortalidad generalizada en repoblaciones de $P$. sylvestris y $P$. nigra en la Sierra de los Filabres (Almería) afectando al menos 10000 ha y extendiéndose a montañas contiguas como la Sierra de Baza (Navarro-Cerrillo et al. 2007). La ausencia de patógenos y la coincidencia con sequías extremas, especialmente en la década de 1990-1999, sugirió que el factor desencadenante del decaimiento era de origen climático.
Comúnmente se ha considerado la hipótesis de un decaimiento secuencial basada en múltiples factores (Manion 1981): (1) "factores de predisposición" que exponen a los árboles a un estrés prolongado (por ejemplo, suelos rocosos y superficiales en sitios xéricos, altas densidades de plantación) y aumentan la susceptibilidad de los árboles frente a (2) estreses, que actúan a corto plazo conocidos como "factores de incitación" (p.ej., sequías extremas), y que dan paso a (3) los "factores de contribución" (p. ej., parásitos), que contribuyen a la mortalidad final del arbolado. A pesar de la aceptación general de este modelo conceptual, el soporte empírico es relativamente débil debido a que los estudios sobre decaimiento inducido por sequía raramente analizan la importancia relativa de todos los factores que están potencialmente implicados así como de sus interacciones. Este es particularmente el caso en los estudios del decaimiento de las repoblaciones forestales mediterráneas (Sánchez-Salguero et al. 2010; 2012 a,b).

En este trabajo se cuantifican los efectos de la sequía sobre el crecimiento y el vigor del arbolado de dos especies de pino $(P$. sylvestris, $P$. nigra) con contrastada vulnerabilidad frente al estrés hídrico en repoblaciones del sureste de la Península Ibérica, utilizando la defoliación como un indicador reciente del vigor (ver Dobbertin, 2005). Los objetivos son: (i) cuantificar los cambios recientes de crecimiento radial; (ii) evaluar si el crecimiento de los individuos más defoliados es más sensible al clima que el de los individuos menos defoliados; (iii) identificar los principales factores abióticos que puedan estar causando el decaimiento forestal; y (iv) cuantificar la relación de estos factores con la defoliación y el crecimiento. Para ello se utilizaron datos climáticos, dendrocronología, evaluaciones visuales de defoliación y datos estructurales tomados en campo. Estos datos se analizaron usando modelos lineales mixtos y logísticos para cuantificar las respuestas del crecimiento y el vigor de estas especies a la estructura del rodal, las condiciones del sitio (intensidad de competencia) y la sequía.

\section{Material y Métodos}

\section{Especies y zona de estudio}

El área de estudio incluye las repoblaciones de $P$. sylvestris (pino silvestre) y $P$. nigra (pino salgareño) situadas en la sierra de Los Filabres ( $37^{\circ} 22^{\prime} \mathrm{N}, 2^{\circ} 50^{\prime} \mathrm{W}$, altitud entre 300-2186 m s.n.m) (Fig. 1). El clima de la zona es mediterráneo continental con tendencia a semiárido, ya que las precipitaciones medias anuales fueron de $320 \mathrm{~mm}$ a $1000 \mathrm{~m}$ de altitud (con un incremento de $+10 \mathrm{~mm}$ por cada $100 \mathrm{~m}$ de altitud ascendidos) (Sierra de los Filabres, en adelante "Filabres") durante el periodo 1940-2009. Las temperaturas medias anuales estimadas a $1000 \mathrm{~m}$ de altitud fueron $13.1^{\circ} \mathrm{C}$ (Sánchez-Salguero et al. 2010). Los suelos dominantes son regosoles y la topografía está caracterizada por pendientes elevadas, habitualmente superiores al 35\% (LUCDEME 2004).

En el área de estudio se repoblaron ambas especies de pinos en la década de 1970-1979 usando semillas del sur y centro de España en el caso de $P$. sylvestris y $P$. nigra, respectivamente. Posteriormente se han realizado tratamientos selvícolas selectivos en dichas repoblaciones (Serrada et al. 2008). Los datos estructurales de la zona de estudio se muestran en las Tablas 1 y 2.

\section{Muestreo y métodos dendrocronológicos}

Se realizó un muestreo estratificado por especie y grados de decaimiento distribuido por la totalidad de repoblaciones presentes siguiendo la malla de inventario sistemático existente para los montes ordenados (IFN II, 1995) (Tabla 2). Los árboles seleccionados correspondían a individuos representativos de cada clase de daños y estaban localizados dentro de la masa, es decir a más de $100 \mathrm{~m}$ del borde de la repoblación. Para cada árbol se estimó su tamaño (diámetro a $1.3 \mathrm{~m}$, altura total) y su defoliación (\%) de copa (Tabla 1) y se tomaron datos adicionales a nivel del sitio. Basándonos en análisis preliminares, los árboles se agruparon en dos clases de decaimiento o categorías de daño atendiendo a su defoliación: árboles con un nivel de defoliación inferior o superior al 50\% de la 


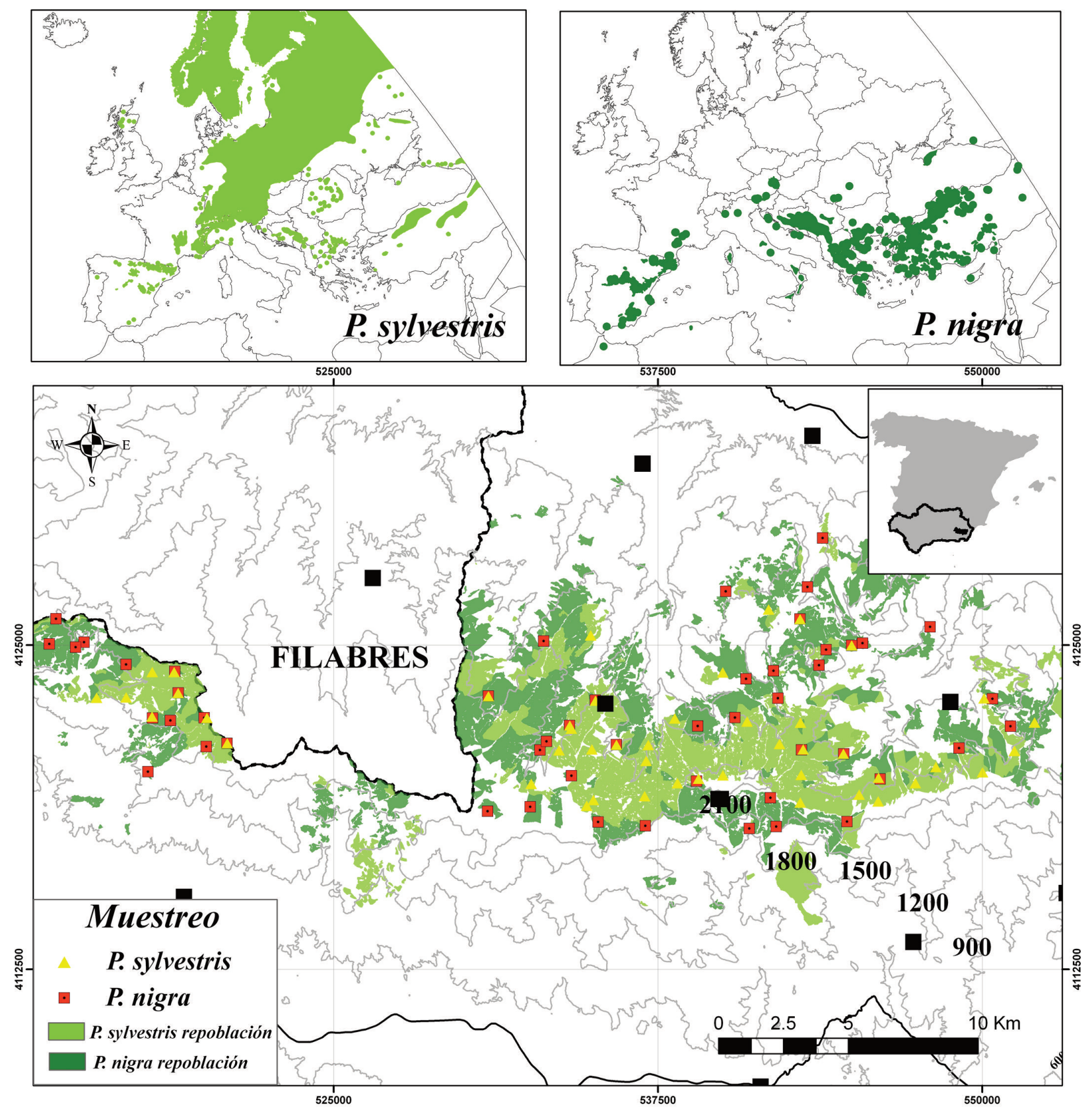

Figura 1. Área de estudio, sitios de muestreo (triángulos y cuadrados rojos) y estaciones meteorológicas próximas (cuadrados negros) situadas en la zona de la Sierra de Los Filabres. Los mapas superiores muestras la distribución de cada especie en Europa y el inferior la zona de estudio en el sudeste de España.

copa, respectivamente, distinguiendo dos clases de daño: árboles con baja defoliación (S, sano; defoliación < $50 \%$ ) o alta defoliación (D, dañados; defoliación $\geq 50 \%$ ) (Dobyshev et al. 2007). Esta hipótesis fue apoyada por los datos fisiológicos de conductancia estomática y potencial hídrico que fueron significativamente menores en árboles defoliados (D) que en los no defoliados (S) en ambas especies (Hernández-Clemente et al. 2011).

Para cada árbol se obtuvo una sección a $1.3 \mathrm{~m}$ de altura que se secó y lijó con lijas de grano sucesivamente más fino hasta distinguir los anillos de crecimiento. Posteriormente las secciones fueron escaneadas y digitalizadas. Se seleccionaron dos radios por sección en direcciones opuestas y perpendiculares a la máxima pendiente de la ladera donde estaba el árbol para evitar la presencia de madera de reacción. Las muestras se dataron visualmente mediante el reconocimiento de anillos característicos (Yamaguchi, 1991). Se midieron los anillos anuales de crecimiento en las muestras ya sincronizadas (180 radios medidos en 90 discos para el periodo 1978-2006) con una resolución de $0.01 \mathrm{~mm}$ usando el sistema de medición semi-automática WinDendro ${ }^{\mathrm{TM}}$ (Regents Co., Canada).
Una vez elaboradas las series brutas de crecimiento se evaluó su datación visual mediante el programa COFECHA (Holmes 1983). Se calculó el incremento de área basal (IAB) usando esta fórmula $\mathrm{I} \mathrm{AB}=\pi\left(R^{2} t-R^{2} t-1\right)$, donde $R$ es el radio del árbol y $t$ es el año de formación del anillo, y asumiendo que las secciones de los troncos eran circulares. Se obtuvieron series medias de IAB para cada individuo, categoría de daño y especie (Sánchez-Salguero et al. 2012b). Finalmente, se construyeron cronologías de índices residuales de crecimiento para cada especie eliminando las tendencias de crecimiento a largo plazo, mediante ajustes de funciones exponenciales negativas, y la autocorrelación temporal anual mediante el programa ARSTAN (Cook 1985). Se calcularon varios estadísticos descriptivos para el intervalo común 1978-2006 (Fritts, 1976) a partir de los anillos de crecimiento (AR1), y las cronologías residuales (MS, rbt) siendo: AR1, la autocorrelación de primer orden, una medida de la similitud del crecimiento de año a año; MS, la sensibilidad media que mide la variabilidad de año a año en el ancho de los anillos consecutivos; y rbt, la correlación media entre árboles que cuantifica la similitud en el crecimiento entre individuos. La señal expresada por la cronología de la población (EPS) con va- 
Tabla 1. Datos usados en los modelos de decaimiento para P. sylvestris (Ps) y P. nigra (Pn).

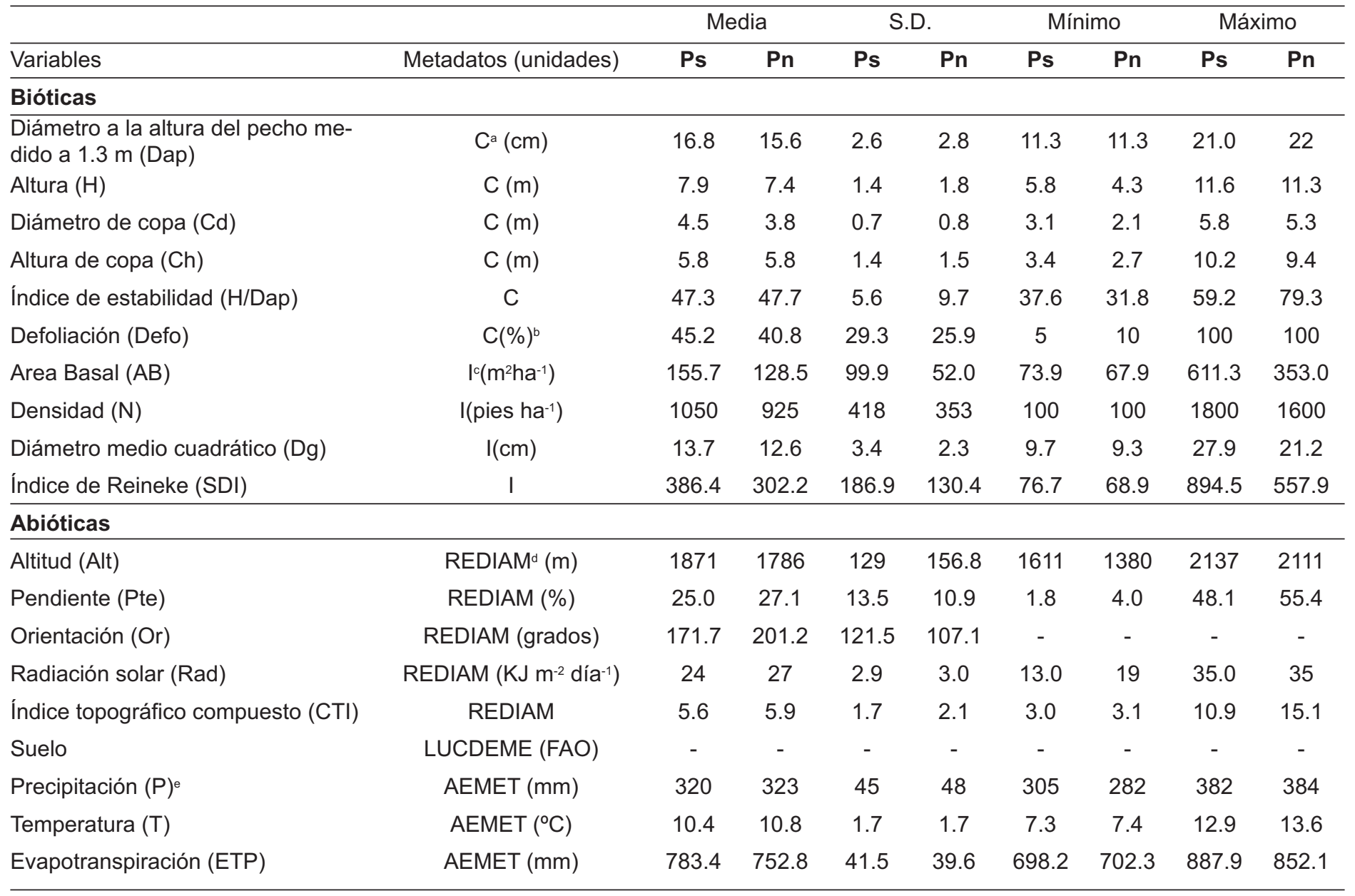

a C: Trabajo de campo realizado en invierno 2006-2007.

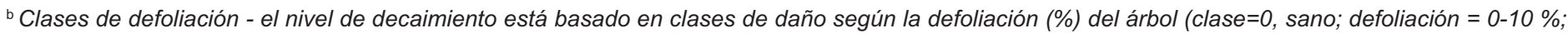
clase 1 = daño leve, defoliación = 11-25\%, clase 2 = árboles con daño moderado a intensa defoliación = 26-60\%; clase $3=$ severamente dañados o muertos, defoliación = 61 - 99\%, clase 4 = árbol muerto, defoliación =100\%) siguiendo a Mueller y Stierlin (1990).

c l: datos procedentes del Inventario Forestal (2007). Todos los árboles fueron muestreados dentro de las parcelas (muestreo sistemático de $1 \times 1 \mathrm{~km}$ ).

${ }^{\mathrm{d}}$ REDIAM (Red de Información Ambiental de Andalucía, Consejería de Medio Ambiente, 2009). Los datos topográficos están basados en un Modelo digital del terreno con una resolución de 10 x 10 metros. Los datos de suelo están basados en la clasificación de FAO.

e Precipitación, Temperatura y Evapotranspiración fueron calculados mensualmente (números desde Enero-1 a Diciembre-12) y para las diferentes estaciones a partir de los datos de 30 estaciones meteorológicas locales (ver Sánchez-Salguero et al. 2010, 2012a,b), AEMET (Agencia Estatal de Meteorología) y REDIAM. Esta información se consiguió para cada parcela en formato raster.

Tabla 2. Datos medios de los árboles muestreados en la Sierra de los Filabres y cuyas series de crecimiento se analizaron mediante métodos dendrocronológicos para árboles sanos (S. defoliación <50\%) y dañados (D. defoliación $\geq 50 \%$ ) considerando las dos especies estudiadas. Se muestran las medias \pm error estándar. Letras diferentes indican diferencias significativas $(P<0.05)$ entre clases de defoliación según el test de Mann-Whitney; su ausencia indica diferencias no significativas.

\begin{tabular}{|c|c|c|c|c|c|c|c|c|}
\hline \multirow{2}{*}{$\begin{array}{c}\text { Categorías de } \\
\text { daño }\end{array}$} & \multicolumn{2}{|c|}{ Número de árboles (radios) } & \multicolumn{2}{|c|}{ Dap (cm) } & \multicolumn{2}{|c|}{ Altura (m) } & \multicolumn{2}{|c|}{ Defoliación (\%) } \\
\hline & P. sylvestris & P. nigra & P. sylvestris & P. nigra & P. sylvestris & P. nigra & P. sylvestris & P. nigra \\
\hline $\mathrm{D}$ & $15(30)$ & $18(36)$ & $15.2 \pm 0.7 b$ & $14.4 \pm 0.5 b$ & $7.3 \pm 0.3$ & $7.1 \pm 0.4$ & $68.0 \pm 6.4 b$ & $63.0 \pm 4.4 \mathrm{~b}$ \\
\hline$S$ & $2.34 \pm 0.18 a$ & $2.4 \pm 0.1 a$ & $0.24 \pm 0.01 b$ & $0.25 \pm 0.01 b$ & $0.61 \pm 0.03 b$ & $0.65 \pm 0.02 b$ & $0.65 \pm 0.03$ & $0.70 \pm 0.02$ \\
\hline$D$ & $2.05 \pm 0.22 b$ & $2.1 \pm 0.2 b$ & $0.32 \pm 0.01 a$ & $0.32 \pm 0.01 a$ & $0.75 \pm 0.02 a$ & $0.71 \pm 0.02 a$ & $0.71 \pm 0.01$ & $0.73 \pm 0.01$ \\
\hline
\end{tabular}

${ }^{1}$ La anchura de anillo fue calculada para el periodo 1978-2006.

${ }^{2}$ Abreviaturas de variables: Dap: diámetro medido a $1.30 \mathrm{~m}$ de altura; MSx: sensibilidad media de la cronologías residuales; AR1: autocorrelación de primer orden de la cronología estándar; rbt: correlación media entre árboles.. 
lores superiores a 0.85 fue considerada como fiable y utilizada en los posteriores análisis de relación crecimiento-clima, siendo EPS una medida de la calidad estadística de la cronología medida en comparación con una cronología perfecta infinitamente replicada (Wigley et al. 1984).

\section{Datos climáticos y variables analizadas}

Los datos climáticos utilizados en este trabajo proceden de la red nacional de estaciones locales con registros largos y continuos de la zona de estudio (AEMET, Agencia Estatal de Meteorología) (Fig. 2 y Tabla 1). Se obtuvo una serie local para cada conjunto de variables climáticas mensuales (temperatura media, precipitación total) usando la subrutina MET del paquete de programas DPL (Dendrochronology Program Library; Holmes 2001). La homogeneidad de los datos climáticos usados se evaluó utilizando la subrutina HOM.

Los datos climáticos y las variables de estación fueron interpolados para cada parcela estudiada usando Sistemas de Información Geográfica (ArcGIS 9.3, ESRI), realizando una interpolación basada en el inverso de la distancia y curvas spline para predecir los datos climáticos mensuales y medias anuales de precipitación, temperatura y evapotranspiración (ETP). Esta última variable se estimó según el método de Thorntwaite (1948) (Tabla 1).

\section{Relación clima-crecimiento}

Las relaciones entre crecimiento radial y clima se evaluaron para cada especie usando las cronologías medias residuales de índices de crecimiento para ambas categorías de daño y las series climáticas de temperatura media y precipitación total basadas en los datos locales. La relación crecimiento-clima se cuantificó calculando coeficientes de correlación de Pearson entre los índices de crecimiento y los datos climáticos mensuales. Esta operación se realizó para todos los árboles de cada especie. El crecimiento radial suele estar también determinado por el clima del año anterior al de formación del anillo (Fritts 1976), por lo que la ventana de correlación clima-crecimiento incluyó desde agosto del año anterior al de crecimiento hasta septiembre del año de formación del anillo. Estos análisis se realizaron usando el programa DENDROCLIM 2002 (Biondi y Waikul 2004).

\section{Análisis estadísticos}

Se comprobó la normalidad y homocedasticidad de todas las variables y los datos de crecimiento mediante tests de Kolmogorov-Smirnov. Una vez comprobados estos aspectos y transformadas las variables correspondientes, se estudiaron todas las variables bióticas y abióticas mediante la aplicación de técnicas de reducción de datos para evitar los problemas de convergencia durante la estimación de parámetros. Se empleó para ello un análisis de componentes principales (ACP) calculado a partir de la matriz de correlaciones entre todas las variables bióticas y abióticas con la defoliación y el crecimiento.

\section{Modelos de decaimiento de copa}

Se usó un modelo logístico para estudiar la probabilidad $(\mathrm{Pr})$ de nivel de daño por cada árbol individual ( $S$, defoliación $<50 \%$; D, defoliación $\geq 50 \%$ ) siguiendo a Bigler et al. (2006) y Lines et al. (2010):

$$
\operatorname{Pr}\left(Y_{i, t}=1 \mid X_{i, t}\right)=1 / 1+\exp \left(X_{i, t} \beta\right)^{-1} \quad \text { [Ecuación 1] }
$$

donde $Y_{i, t}$ es el estado del árbol $i$ en el tiempo $t(Y=0$ árbol sano (S), $Y=1$ árbol dañado o muerto (D)), $\operatorname{Pr}\left(Y_{i, t}=1 \mid X_{i, t}\right)$ es la probabilidad de daño de copa en el intervalo $[0,1]$ y $X_{i, t} \beta$ es una combinación lineal de las variables independientes $(X)$ y los coeficientes de regresión $(\beta)$. Para seleccionar el mejor modelo logístico, se realizaron la selección de variables minimizando los valores de AIC (Burnham y Anderson 2002; Alenius et al. 2003).

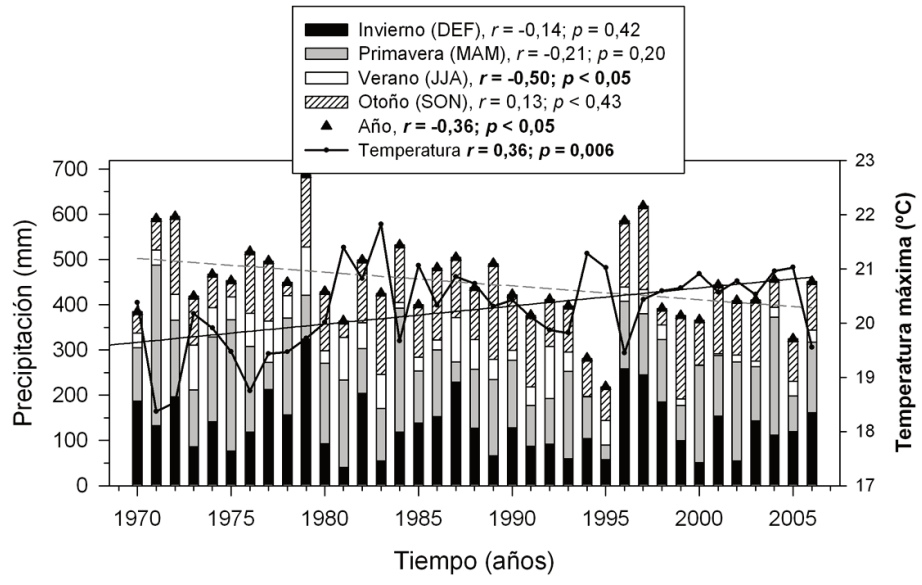

Figura 2. Evolución reciente de la precipitación estacional (barras) y anual (triángulos) y de la temperatura media de las máximas (línea con puntos) en la Sierra de Filabres basada en la serie local climática elaborada (se muestran los coeficientes de correlación y su probabilidad asociada para cada estación del año y para la serie anual; los coeficientes significativos se indican en negrita). Las regresiones lineales indican tendencias opuestas para la precipitación anual (línea discontinua) y para la temperatura máxima (línea continua).

\section{Modelos de crecimiento radial}

Se calcularon modelos de crecimiento radial (IAB) para determinar la evolución de esta variable durante el proceso de decaimiento y para evaluar si existían diferencias entre especies y categorías de daño. Dado que la distribución de IAB estaba sesgada se utilizó como variable respuesta el logaritmo del IAB. Se usaron modelos lineales mixtos para analizar el efecto de las variables independientes sobre el crecimiento de árboles en ambas especies para las dos clases de daño. La estructura general de los modelos fue:

$$
Y_{i j}=\mu+X_{i} \beta_{i}+Z_{i j}+\xi_{i j}
$$

[Ecuación 2]

donde $Y_{i j}$ es el vector que contiene los valores de log(IAB) para el árbol $i$ y el año $j, \mu$ es el intercepto, $X_{i}$ corresponde a la matriz de efectos fijos (p. ej. tamaño del árbol, variables topográficas, etc), $\beta_{i}$ es el vector asociado con los parámetros de los efectos fijos, $Z_{i j}$ es la matriz de efectos aleatorios (año, árbol) y $\xi_{i j}$ es el término de error aleatorio para el árbol $i$ y el año $j$. Para seleccionar el mejor modelo, se realizaron la selección de variables minimizando los valores de AIC (Burnham y Anderson 2002). Finalmente, se reservaron un $10 \%$ de las parcelas iniciales para la validación de los modelos. Todos los análisis fueron realizados con SAS v. 9.1.3 (SAS Corporation, Cary, NC, USA).

\section{Resultados}

\section{Análisis climático}

Detectamos un aumento significativo de la temperatura media máxima en el área de estudio y descensos significativos de las precipitaciones primaverales y otoñales durante el siglo XX (Fig. 2). La estacionalidad de las precipitaciones se ha modificado a lo largo del siglo XX, pasando de una distribución fundamentalmente primaveral a precipitaciones otoñales cada vez más importantes (Fig. 2). Como consecuencia de este cambio en la distribución estacional de precipitaciones, la aridez a comienzo del año ha aumentado significativamente, lo que indica un mayor déficit hídrico que fue patente en las sequías extremas de 1994-1995, 1999 y 2005, que representan el 5\% de los valores más bajos de déficit hídrico desde 1950.

\section{Variables relacionadas con el decaimiento}

Nuestros análisis correlacionales con crecimiento y defoliación indican que estresores relacionados con la competencia entre árboles vecinos y el clima, en concreto eventos extremos y recurren- 
tes de sequía, son los factores de predisposición más importantes del decaimiento estudiado. El diagrama de dispersión del análisis de componentes principales muestra un grupo representativo de factores relacionados con la competencia (p.ej., diámetro de copa) y otro relacionado con variables climáticas (p.ej., evapotranspiración potencial y temperaturas máximas) como posibles factores causantes del decaimiento de las dos especies estudiadas de pino (Fig. 3).

\section{Patrones de crecimiento y clima}

Los árboles sanos mostraron mayor diámetro medido a $1.3 \mathrm{~m}$ que los defoliados (Tabla 2). La media de anchura de anillo para el período de 1978-2006 fue mayor para árboles sanos que para los dañados (D) en ambas especies, y la sensibilidad media (MSx) y la autocorrelación de primer orden (AR1) de las series de anchura de anillos fueron mayores en árboles dañados que en sanos para las dos especies (Tabla 2), mientras que la correlación entre árboles $(r b t)$ no mostró diferencias entre clases de daño dentro de cada especie.

Las series de incremento de área basal mostraron tendencias similares de crecimiento en ciertos años ya sea como aumentos (1992, 1997) o como declives, que fueron especialmente bruscos en 1994-1995, 1999 y 2005 coincidiendo con años de acusado déficit hídrico (Fig. 4). Desde finales de la década de 1990-1999 se apreciaron divergencias de crecimiento entre árboles con defoliación reciente superior o inferior al $50 \%$ de la copa, aunque en el caso de $P$. sylvestris las divergencias se observaron desde el comienzo de las series. La divergencia de crecimiento entre árboles poco y muy defoliados ha sido mucho mayor en el caso de $P$. sylvestris que en el de $P$. nigra.

El crecimiento radial en las dos especies estudiadas presentó una relación significativa y positiva con las precipitaciones de mayo y junio del año de formación del anillo, y negativa con las temperaturas del mismo periodo (Fig. 5). El crecimiento de ambos pinos estuvo relacionado de forma positiva con la precipitación de enero y marzo. El crecimiento de los árboles más defoliados respondió más al estrés hídrico que el de los árboles menos defoliados para ambas especies, siendo la diferencia mayor en el caso de $P$. sylvestris que en el de $P$. nigra (resultados no mostrados).

\section{Modelo de decaimiento}

En primer lugar se realizó un análisis discriminante con los factores bióticos y abióticos para agrupar según las categorías de defoliación definidas, obteniendo un ajuste para el límite de defoliación del $50 \%(p=0.01)$, clasificándose correctamente el 93\% de los casos agrupados originales y siendo dicha clasificación significativa.

Posteriormente se elaboró el modelo de decaimiento para estas dos categorías. Las variables que mejor explican el decaimiento en base en los modelos de defoliación de copa para ambas especies fueron las relacionadas con la competencia entre árboles (p.ej. diámetros de tronco y de copa) y la condiciones climáticas del mes de junio. La temperatura y precipitación de junio condicionan el desarrollo del arbolado, por tanto los efectos de las sequía extremas en ese periodo pueden actuar como factores de incitación al decaimiento. Además para $P$. sylvestris se asoció el decaimiento con la evapotranspiración de verano (Tabla 3).

El punto óptimo de corte para el modelo logístico fue para la clasificación de modelo a 0.50 (es decir, los árboles se clasifican como sano "S" o dañado "D" cuando la probabilidad de daño es inferior o superior a 0.50 , respectivamente). El modelo de $P$. nigra predijo correctamente el $92 \%$ de los casos y el modelo de $P$. sylvestris predijo correctamente el $91 \%$ de los casos.

\section{Modelos de crecimiento}

El modelo de crecimiento seleccionado para $P$. nigra detectó que los factores más importantes en el crecimiento fueron los diámetros del tronco y de la copa, que ejercen efectos positivos sobre
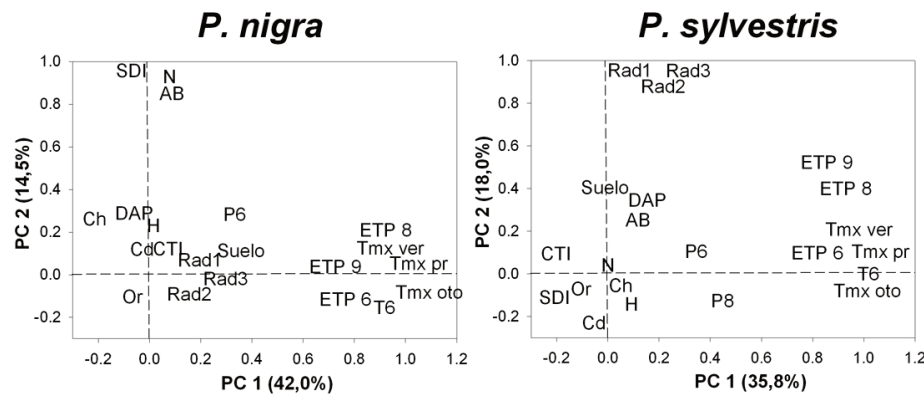

Figure 3. Diagrama de dispersión de los componentes principales obtenidos a partir de una matriz de correlación entre las variables independientes para ambas especies. El porcentaje de varianza explicada por los dos primeros ejes principales se muestra. Los códigos de letras para cada variable son los mismos que los usados en la Tabla 1: N. densidad; SDI. índice de Reineke; $A B$. área basal; Ch. altura de copa; Cd. diámetro de copa; H. altura; Dap. diámetro normal; Rad. radiación solar (1-equinocio de primavera. 2- solsticio de verano. 3-equinocio de otoño); Or. orientación; Suelo. tipo de suelo según clasificación FAO; CTI. índice topográfico compuesto; P. precipitación (desde 1-Enero a 12-Diciembre); ETP. evapotranspiración potencial (desde 1-Enero a 12-Diciembre); Tmx pr. temperatura media de las máximas en primavera; Tmx ver. temperatura media de las máximas en verano; Tmx oto. temperatura media de las máximas en otoño.

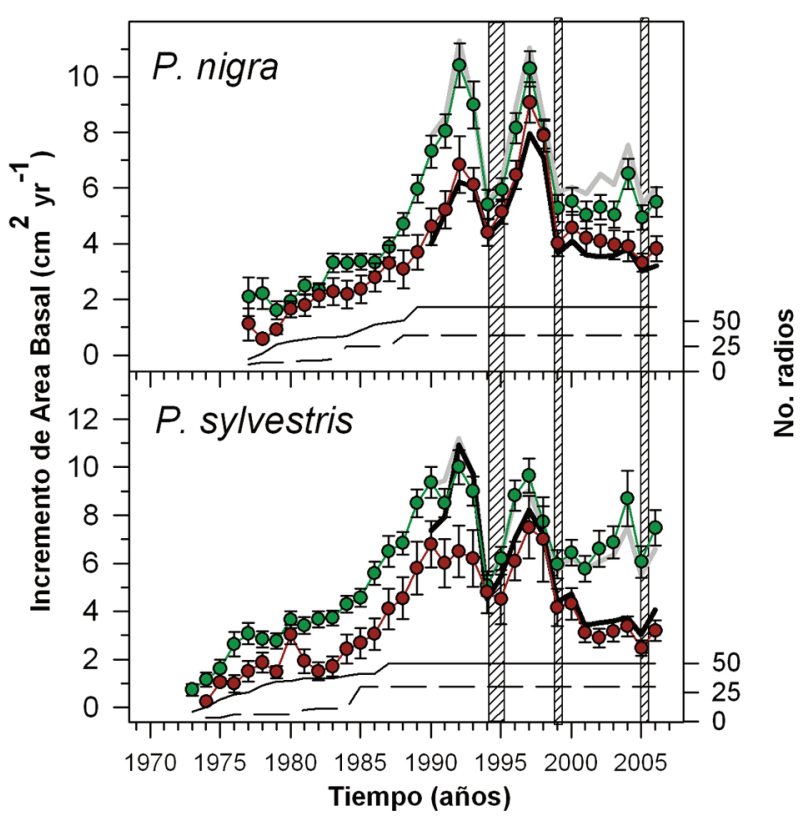

Figura 4. Tendencias del incremento de área basal (IAB) observado (líneas con puntos) y predicho (líneas) en las dos especies estudiadas en Filabres agrupadas por categorías de daño; árboles con baja defoliación (S. sano; defoliación < 50\%) (línea verde) o alta defoliación (D. dañados; defoliación $\geq 50 \%$ ) (línea roja). Las líneas continuas (gris. árboles sanos; negra. árboles dañados) corresponden con el incremento de área basal predicho por los modelos mixtos para el periodo 1990-2006. Las barras verticales rayadas indican los años de mayor déficit hídrico durante la segunda mitad del s. XX (1994, 1995, 1999 y 2005). La escala de la derecha muestra el número anual de radios medidos para árboles con baja (líneas continuas) y alta defoliación (líneas discontinuas). Las barras de error corresponden al error estándar.

el incremento en área basal (IAB), pero el diámetro de la copa sólo fue relevante en el caso de los árboles dañados (Tabla 4). La precipitación de junio tenía efectos positivos sobre el crecimiento en ambos niveles de daño; y la temperatura de junio mostró efectos negativos significativos sobre IAB sólo en los árboles dañados (Tabla 4). La orientación estaba relacionada negativamente y de manera significativa con el IAB en las dos categorías de árboles, pero el suelo mostró efectos negativos significativos sólo en los árboles sanos. El factor tiempo fue positivo, lo que indica la tendencia positiva del IAB. 
Tabla 3. Modelo logístico seleccionado para predecir el decaimiento en $\mathrm{P}$. nigra y P. sylvestris en Filabres basado en la Ecuación (1) (tabla adaptada de Sánchez-Salguero et al. 2012b). Abreviaturas de las variables en la Tabla 1.

\begin{tabular}{llrrr}
\hline Especies & \multicolumn{1}{c}{ Variable } & $\begin{array}{c}\text { Parámetros } \\
\text { (Error estándar) }\end{array}$ & AIC & R $^{2}$ Nagelkerke \\
\hline P. nigra & $\left(\beta_{0}\right)$ Constante & $-47.561(18.128)^{*}$ & 18.517 & 0.805 \\
& $\left(\beta_{1}\right)$ Cd & $-2.636(1.122)^{*}$ & & \\
& $\left(\beta_{2}\right)$ Tjunio & $3.268(1.170)^{*}$ & & \\
& $\left(\beta_{3}\right)$ SDI & $0.023(0.010)^{*}$ & & \\
& $\left(\beta_{4}\right)$ Pjunio & $-0.861(0.383)^{*}$ & & \\
\hline P. sylvestris & $\left(\beta_{0}\right)$ Constante & $16.887(2.7185)^{*}$ & 31.493 & 0.815 \\
& $\left(\beta_{1}\right)$ Dap & $-0.648(0.363)^{*}$ & \\
& $\left(\beta_{2}\right)$ Tjunio & $0.028(0.646)^{*}$ & & \\
& $\left(\beta_{3}\right)$ ETPverano & $1.425(1.131)^{*}$ & \\
& $\left(\beta_{4}\right)$ Pjunio & $-0.637(0.440)^{*}$ & \\
& $\left(\beta_{5}\right)$ SDI & $0.008(0.004)^{*}$ & \\
\hline
\end{tabular}

Nota: ES- Error estándar. $\beta_{0}$. valor Constante; $\beta_{\mathrm{i}}$. Coeficientes estimados para el modelos; Cd. Diámetro de copa; Tjunio. Temperatura de Junio; SDI. Ídice de Reineke; Pjunio. Precipitación de Junio; Dap. diámetro a 1.30 m; ETPverano. Evapotranspiración de verano; * nivel de significación a $p<0.05$.

En cuanto al modelo seleccionado para $P$. sylvestris mostró que el diámetro del tronco se relacionaba positiva y significativamente con el IAB en ambas categorías de daño (Tabla 4). La intensidad de la competencia mostró efectos negativos significativos sobre el IAB sólo en los árboles dañados (Tabla 4). La pendiente mostró efectos negativos significativos sobre $I A B$ sólo en los árboles sanos, pero el suelo se correlacionó negativamente con el IAB de ambos niveles de daño. En cuanto a las variables del clima, las precipitaciones de junio y la evapotranspiración potencial del verano ejercieron efectos significativos positivos o negativos, respectivamente, sobre el IAB (Tabla 4). Como era de esperar, las variables climáticas mostraron efectos más intensos sobre el IAB en los árboles dañados que en los sanos (Tabla 4). Todos los modelos mostraron predicciones aceptables atendiendo al porcentaje de casos correctamente predichos $(P$. sylvestris, árboles sanos $=65 \%$, árboles dañados $=88 \% ; P$. nigra, árboles sanos $=56 \%$, árboles dañados $=66 \%$ ).

\section{Discusión}

Las repoblaciones de $P$. sylvestris y $P$. nigra de la Sierra de los Filabres están experimentando un proceso grave y acelerado de decaimiento que afecta de manera generalizada a ambas especies, pero notablemente a $P$. sylvestris, especie que muestra el mayor nivel de defoliación reciente (Navarro-Cerrillo et al. 2007, Sánchez-Salguero et al. 2012 a,b). El hecho de que se trate de procesos de decaimiento sin presencia de patógenos primarios directamente implicados y que afecten a diversas especies de pinos y de manera contrastada en localidades diferentes sugiere que el estrés climático junto con la excesiva competencia, mediada por la falta de tratamientos silvícolas como talas o aclareos, son los principales responsable de la pérdida de vigor, la defoliación y el declive del crecimiento radial.

Concretamente, el estrés hídrico inducido por sequías extremas parece ser el principal desencadenante climático del episodio de decaimiento descrito por diversas razones. Primero, $P$. sylvestris es la especie más afectada, lo que concuerda con su mayor vulnerabilidad a episodios de embolia inducidos por estrés hídrico en comparación con P. nigra (Martínez-Vilalta et al. 2004). Segundo, las zonas más afectadas son las que muestra menor disponibilidad hídrica potencial lo que indica que la respuesta del crecimiento y la mortalidad al estrés climático van a estar muy condicionadas por las condiciones locales (Lloret y Siscart 1995; Martínez-Vilalta y Piñol 2002). Tercero, la divergencia en el crecimiento radial entre árboles poco defoliados y muy defoliados en el caso de $P$. sylvestris se ha acentuado después de sequías extremas (1994-1995, 1999). Cuarto, el crecimiento de $P$. sylvestris en la zona de estudio depende en gran medida de la precipitación recibida en mayo y junio, meses que han mostrando un notable descenso de precipitación (Fernández-Cancio et al. 2011; Camarero et al. 2012). Las razones expuestas sugieren que las sequías extremas de 1994-1995 y 1999 , junto con las condiciones de alta competencia desencadenaron y predispusieron (sensu Manion 1981), respectivamente, al decaimiento de las repoblaciones de pinos. La menor resistencia al estrés hídrico de $P$. sylvestris frente a $P$. nigra y las condiciones climáticas mediterráneas continentales con tendencia a la aridez de Filabres también propiciaron el decaimiento citado.

Diversos estudios han encontrado que la mayor variabilidad climática de la segunda mitad del siglo XX ha condicionado en gran medida el crecimiento de poblaciones ibéricas de árboles (Manrique Menéndez y Fernández-Cancio 2000; Tardif et al. 2003; Andreu et al. 2007). La inestabilidad climática reciente es particularmente notable en el sudeste ibérico ya que las sequías extremas de 19941995 y 1999 no parecen haber tenido precedentes similares durante el siglo XX en la zona de estudio. A pesar de que los niveles elevados de defoliación y mortalidad se observaron a partir del año 2001, la divergencia de crecimiento radial entre árboles según su defoliación comenzó a hacerse patente a partir de las sequías de 1994-1995 e incluso antes, y dicha divergencia parece ser ya irreversible en el caso de $P$. sylvestris. Los descensos bruscos de crecimiento de 1994-1995 y 1999 en las repoblaciones de $P$. sylvestris y $P$. nigra en Filabres parecen excepcionales en el contexto de al menos los últimos 30 años.

El menor crecimiento radial de individuos más defoliados de $P$. sylvestris podría explicarse por la mayor pérdida de conductividad hidráulica debida a embolia del xilema de esta especie frente a $P$. nigra ya que el diseño del muestreo redujo en gran medida las diferencias locales de hábitat entre especies que podrían afectar a la distinta disponibilidad de agua en el suelo o a la exposición a

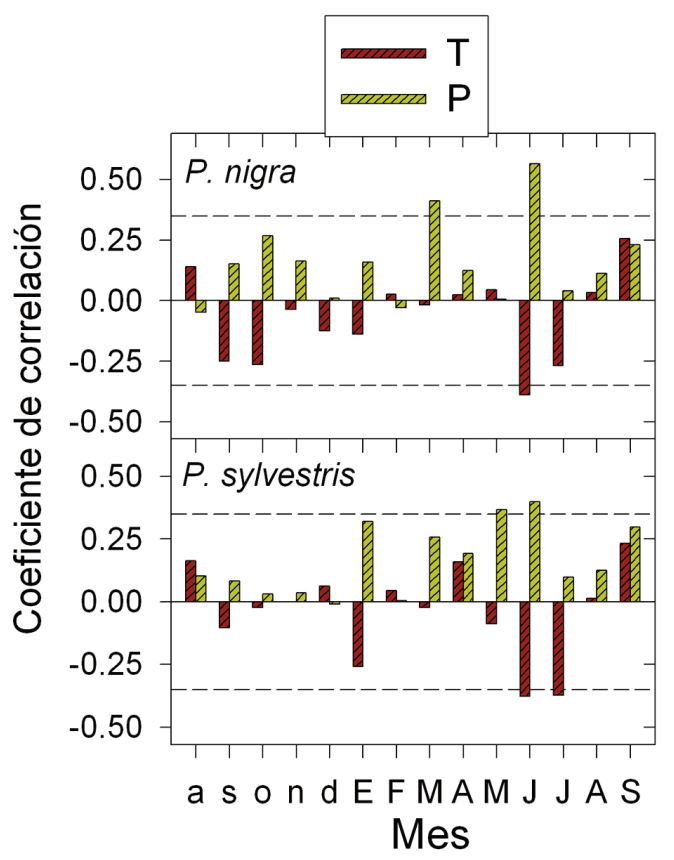

Figura 5. Relaciones entre clima y crecimiento radial (índices residuales de crecimiento) para todas las series de las dos especies de pino estudiadas en las Sierra de Filabres. Las variables climáticas mensuales usadas fueron la temperatura media ( $T$-barras rojas-) y la precipitación acumulada ( $P$ barras verdes-). Se correlacionó el crecimiento con variables climáticas del año previo (meses abreviados con letras minúsculas) y actual o de formación del anillo (meses abreviados con letras mayúsculas). Las líneas discontinuas horizontales indican el nivel de significación $(P<0.05)$. 
Tabla 4. Efectos fijos para el modelo mixto de incremento de área basal en P. nigra y P. sylvestris basado en la Ecuación (2) (ver texto) para árboles sanos (S. defoliación <50\%) y dañados (D. defoliación $\geq 50 \%$ ). Abreviaturas de las variables como en la Tabla 1.

\begin{tabular}{cccccc}
\hline Especie & Efectos fijos & \multicolumn{2}{c}{ Estimador } & \multicolumn{2}{c}{ Error Estándar } \\
\hline & & $\begin{array}{c}\text { Árboles } \\
\text { sanos }\end{array}$ & $\begin{array}{c}\text { Árboles } \\
\text { dañados }\end{array}$ & $\begin{array}{c}\text { Árboles } \\
\text { sanos }\end{array}$ & $\begin{array}{c}\text { Árboles } \\
\text { dañados }\end{array}$ \\
\hline P. nigra & Intercepto & 0.9693 & 2.1950 & 0.3111 & 0.4152 \\
& Tiempo & 0.0229 & 0.0236 & 0.0093 & 0.0094 \\
& Dap & 0.0028 & 0.0025 & 0.0005 & 0.0005 \\
& Cd & 0.0001 & 0.1218 & 0.0195 & 0.0195 \\
& Tjunio & -0.0343 & -0.1227 & 0.0185 & 0.0275 \\
& SDI & -0.0001 & -0.0006 & 0.0001 & 0.0002 \\
& Pjunio & 0.0135 & 0.0226 & 0.0056 & 0.0059 \\
& Suelo & -0.1268 & -0.0560 & 0.0404 & 0.0320 \\
& Orientación & -0.0009 & -0.0003 & 0.0001 & 0.0001 \\
\hline sylvestris & Intercepto & -6.6148 & 7.1138 & 2.7561 & 1.5742 \\
& Tiempo & 0.0118 & 0.0305 & 0.0047 & 0.0110 \\
& Dap & 0.0467 & 0.0367 & 0.0095 & 0.0119 \\
& SDI & -0.00001 & -0.0006 & 0.00007 & 0.0003 \\
& Pendiente & -0.0001 & -0.0001 & 0.0003 & 0.0033 \\
& Suelo & -0.3351 & -0.2931 & 0.1191 & 0.0877 \\
& Tjunio & -1.7183 & -2.3416 & 0.2738 & 1.1439 \\
& Pjunio & 0.0142 & 0.3479 & 0.0055 & 0.1289 \\
& ETPverano & -0.2851 & -0.3731 & 0.0474 & 0.1478 \\
\hline & & & & &
\end{tabular}

temperaturas extremas. En Filabres, la disponibilidad hídrica a finales de primavera parece determinante para la formación de madera ya que es en estos meses cuando la tasa de crecimiento radial suele ser máxima, al menos en zonas de montaña similares en el caso de P. sylvestris (Camarero et al. 1998). Además, similares relaciones crecimiento-clima se han descrito para otros bosques de $P$. sylvestris sometidos también a cierto estrés hídrico estival (Gutiérrez 1989; Rigling et al. 2003; Camarero et al. 2012). En el caso de $P$. nigra sólo encontramos una relación positiva con la precipitación de mayo sugiriendo una xilogénesis distinta respecto a $P$. sylvestris.

Hemos mostrado cómo el descenso brusco en la disponibilidad hídrica en el suelo reduce en general el vigor y crecimiento radial del árbol pero aumenta su respuesta al estrés hídrico dentro de la misma especie. En casos extremos se ha mostrado cómo un estrés hídrico prolongado puede conducir incluso a que aparezcan relaciones negativas entre la temperatura primaveral y el crecimiento radial (Rigling et al. 2003). Dado que las repoblaciones muestran una menor variabilidad genética que los bosques naturales, asumimos que la respuesta diferencial del crecimiento de árboles con vigor contrastado al estrés hídrico puede venir condicionada por diferencias locales de hábitat y competencia entre individuos de la misma especie que condicionen su disponibilidad hídrica (Fernández-Cancio et al. 2011). Por ello, las zonas cuyos suelos presentan escasa retención de agua, como el caso de zonas muy defoliadas de $P$. sylvestris en Filabres, pueden actuar como factores de predisposición frente al decaimiento inducido posteriormente por sequías extremas (Bigler et al. 2006).

Para predecir el decaimiento diversos trabajos han usado como indicador el descenso del incremento de área basal (Duchesne et al. 2003). Por lo tanto, la tendencia del crecimiento radial de un árbol se puede utilizar como un indicador que precede a la aparición de síntomas visibles de defoliación en copa y así servir para una evaluación temprana del vigor del arbolado (Bigler y Bugmann 2003). Varios estudios además han confirmado previamente esta relación en los fenómenos de mortalidad y decaimiento forestales (Dobbertin 2005; Dobyshev et al. 2007; Linares et al. 2012). Otros estudios han demostrado una correlación significativa entre la an- chura de los anillos de crecimiento de los árboles y el grado de defoliación de las copas en varias especies de toda Europa (Juknys et al. 2003; Dobbertin 2005). Los modelos de decaimiento desarrollados representan una valiosa contribución a las directrices sobre las técnicas de manejo forestal en las primeras etapas de gestión de una masa regular repoblada y podrían incorporarse en los planes de gestión futuros. Al mismo tiempo, la importancia de la gestión forestal, con la adecuada selvicultura, a través de los tratamientos orientados a adecuar la espesura de las masas, y las actividades de aprovechamiento sostenible podrían ser utilizadas para mitigar los efectos del cambio climático en repoblaciones de alta densidad muy abundantes en zonas de España con cierto estrés hídrico (Millar et al. 2007; Gómez-Aparicio et al. 2011).

Dentro de esta relación directa entre la defoliación y el crecimiento en los árboles y en el caso de bosques o rodales mostrando elevados niveles de competencia cabe indicar que los árboles más defoliados fueron los más propensos a presentar mayor mortalidad, especialmente en el caso de $P$. sylvestris. Resultados similares con estudios sobre el efecto de la competencia en la supervivencia de los árboles se han mostrado en Austria (Monserud y Sterba 1999), Noruega (Eid y Tuhus 2001), Finlandia (Alenius et al. 2003) y España (Bravo-Oviedo et al. 2006; Jump et al. 2006; Galiano et al. 2010).

Nuestros resultados sugieren que el crecimiento del pino silvestre está fuertemente limitado por la relación entre la disponibilidad de agua y la demanda atmosférica (Martínez-Vilalta et al. 2008), ya que la evapotranspiración potencial fue una variable clave en los modelos de decaimiento y crecimiento, siendo mayor el impacto en los árboles sanos que en los dañados (Carnicer et al. 2011) (Tabla 4). En general, el efecto de la temperatura sobre el crecimiento de ambas especies fue negativo, pero este efecto debe evaluarse respecto a la disponibilidad de agua ya que durante años húmedos las temperaturas más altas pueden provocar un mayor crecimiento, mientras que lo contrario puede ocurrir durante los años secos. El aumento de la demanda hídrica inducido por temperaturas más elevadas puede explicar el impacto de la evapotranspiración de verano sobre el crecimiento radial (Vilà-Cabrera et al. 2011).

Nuestro trabajo confirmó la asociación esperada entre el decaimiento -aumento de la defoliación y mortalidad-, las condiciones climáticas de primavera y verano y la competencia previamente observada en otros bosques mediterráneos (Linares et al. 2009, 2010, 2012). Nuestros resultados proporcionan un apoyo adicional a los estudios previos en decaimiento forestal que muestran una alta vulnerabilidad a las sequías severas en los límites de tolerancia climáticos más xéricos y en condiciones de alta competencia (Lloret 2012), por ejemplo en casos de bosques no gestionados o con elevadas densidades y niveles de competencia estudiados en ambas especies ( $P$. sylvestris: Martínez-Vilalta y Piñol 2002; Galiano et al. 2010, 2011; Martínez-Vilalta et al. 2012; P. nigra: Palahí y Grau 2003; Linares y Tiscar 2010; Candel-Pérez et al. 2012).

Este trabajo sugiere además que la relación entre estresores implicados en el decaimiento es compleja y puede involucrar a una amplia gama de variables (Sánchez-Salguero 2012) (Fig. 6). El proceso de decaimiento puede ser visto como un resultado de una serie de eventos en cadena con las primeras sequías extremas (1994-1995) actuando como factores de incitación de forma sinérgica a factores previos de predisposición como la estructura de la masa y la elevada competencia (Fig. 6). En el sudeste de España, las repoblaciones de las dos especies de pino estudiadas no han sido capaces de aclimatarse a las nuevas condiciones más secas y calurosas durante el siglo $X X$, especialmente en el caso de repoblaciones de pino silvestre que se encuentran cerca de los bosques más meridionales de la distribución natural de la especie. Nuestros resultados proporcionan apoyo adicional a los estudios previos que muestran la alta vulnerabilidad frente a la sequía de los bosques de coníferas situados cerca de los límites geográficos meridionales de distribución y en condiciones climáticas xéricas como el pino silvestre o el abeto (Macías et al. 2006). 


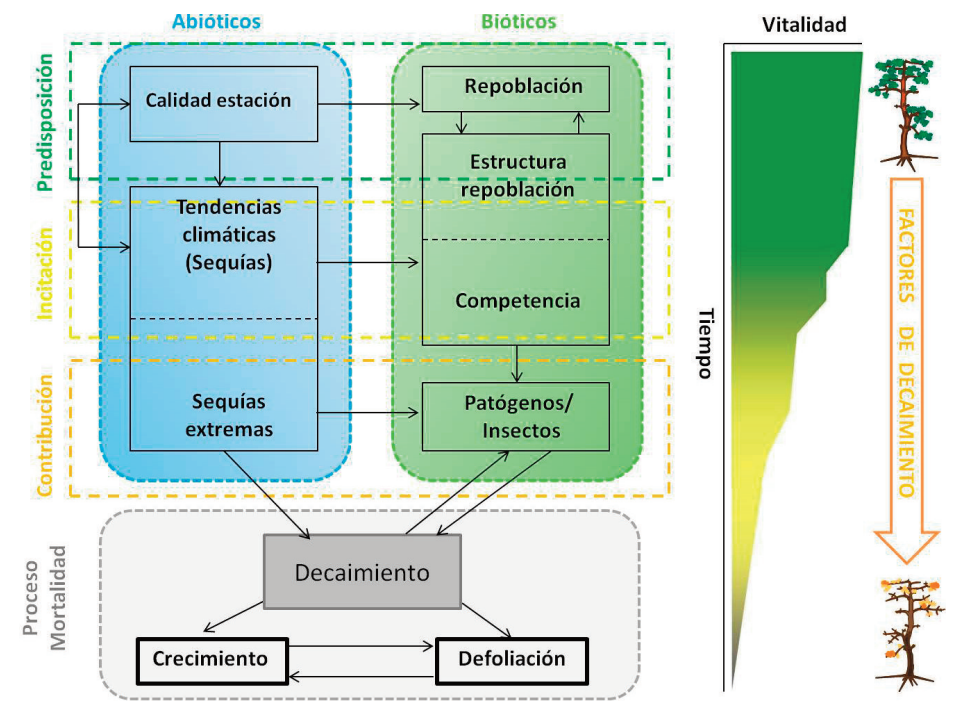

Figura 6. Modelo conceptual de decaimiento forestal en repoblaciones forestales de pinos que soportan múltiples factores abióticos y bióticos de estrés (adaptado de Sánchez-Salguero 2012).

\section{Conclusiones}

Los datos climáticos regionales muestran un incremento de la aridez en las sierras del sudeste ibérico debido al aumento de las temperaturas máximas y al descenso de las precipitaciones primaverales y otoñales durante la segunda mitad del s. XX. El índice de sequía y el déficit hídrico estimado indican que las sequías de 1994-1995 y 1999 no tuvieron precedentes similares en el siglo XX. Los datos dendrocronológicos confirman que las repoblaciones con mayor grado de defoliación como los pinares de $P$. sylvestris de la Sierra de los Filabres muestran una pérdida reciente de vigor relacionada con un descenso del crecimiento radial en respuesta a las sequías de 1994-1995 y 1999 y a condiciones previas de elevada competencia. La reducción de la disponibilidad hídrica desde finales del siglo XX ha causado una reducción más intensa del crecimiento y una mayor aumento de la defoliación de la especie más vulnerable a la embolia por estrés hídrico $(P$. sylvestris) respecto a la más resistente ( $P$. nigra). Los modelos de decaimiento desarrollados para $P$. nigra y $P$. sylvestris revelan que la probabilidad de daño de un árbol está condicionada por su tamaño del árbol y por la competencia que experimenta así como por factores de estrés climático. Las categorías de daño establecidas usando un umbral de defoliación del $50 \%$ son útiles para predecir las etapas del decaimiento y para elaborar modelos de decaimiento que puedan ser empleados por los gestores forestales.

\section{Agradecimientos}

Este estudio se ha realizado en el marco de los proyectos "Estudios de modelos de predicción de procesos de decaimiento en masas de Pinus sylvestris L. y Pinus nigra Arnold. en la Sierra de los Filabres a partir de datos dendrocronológicos" (Convenio Consejería de Medio Ambiente-Grupo PAI 360), DIVERBOS (CGL2011-30285-C02-02), SUM2008-00004-C03.01 (INIA), ANASINQUE (PGC2010-RNM-5782), CEXTREME; FP7-ENV-2008-1226701 y por el Proyecto INIA-RTA (RTA2010-00065-00-00) programa FPU (AP2007-04747) del Ministerio de Educación. Agradecemos a J. Bautista y A. Herrero su ayuda en el trabajo de campo, así como a los técnicos de la Consejería de Medio Ambiente, dirección del P.N. de Sierra de Baza y grupo de sanidad forestal de EGMASA por el apoyo logístico, material y de personal (Migüi y Almudena) para realizar este estudio y a J. Lara por su ayuda en laboratorio. El primer autor agradece a todos los miembros del "Laboratory of Tree-Ring Research" de la Universidad de Arizona y a P. Sheppard por sus útiles comentarios. JJC agradece el apoyo de la Fundación ARAID.

\section{Referencias}

Alenius, V., Hökkä, H., Salminen, H., Jutras, S. 2003. Evaluating estimation methods for logistic regression in modeling individual-tree mortality. En: Amaro, A., Reed, D. and Soares, P. (eds). Modelling forest systems. CABI Publishing, Wallingford, UK.

Allen, C.D., Macalady, A.K., Chenchouni, H., Bachelet, D., McDowell, N., Vennetier, M., Kitzberger, T., Rigling, A., Breshears, D.D., Hogg, E.H., Gonzalez, P., Fensham, R., Zhang, Z., Castro, J., Demidova, N., Lim, J.H., Allard, G., Running, S.W., Semerci, A., Cobb, N. 2010. A global overview of drought and heat-induced tree mortality reveals emerging climate change risks for forests. Forest Ecology and Management. 259:660-684.

Andreu, L., Gutiérrez, E., Macias, M., Ribas, M., Bosch, O., Camarero, J.J. 2007. Climate increases regional tree-growth variability in Iberian pine forests. Global Change Biology 13:804-815.

Bigler. C., Bugmann. H. 2003. Growth-dependent tree mortality models based on tree rings. Canadian Journal of Forest Research. 33:210-221.

Bigler, C., Bräker, O.U, Bugmann, H., Dobbertin, M., Rigling, A. 2006. Drought as an inciting mortality factor in Scots pine stands of the Valais, Switzerland. Ecosystems 9:330-343.Bigler, C., Bugmann, H. 2003. Growth-dependent tree mortality models based on tree rings. Canadian Journal of Forest Research. 33:210-221.

Biondi, F., Waikul, K. 2004. DENDROCLIM2002: a C++ program for statistical calibration of climate signals in tree-ring chronologies. Computers and Geosciences 30:303-311.

Bonan, G.B. 2008. Forests and climate change: Forcings, feedbacks, and the climate benefits of forests. Science 320:1444-1449.

Bravo-Oviedo, A., Sterba, H., del Río, M., Bravo, F. 2006. Competition-induced mortality for Mediterranean Pinus pinaster Ait. and P. sylvestris L. Forest Ecology and Management 222:88-98.

Burnham, K.P., Anderson, D.R. 2002. Model Selection and Multimodel Inference: a Practical Information-Theoretic Approach. Springer, Heidelberg, Germany.

Camarero, J.J., Guerrero-Campo, J., Gutiérrez, E. 1998. Tree-ring growth and structure of Pinus uncinata and Pinus sylvestris in the Central Spanish Pyrenees. Arctic and Alpine Research 30:1-10.

Camarero, J.J., Bigler, C.J., Linares, J.C., Gil-Peregrín, E. 2011. Synergistic effects of past historical logging and drought on the decline of Pyrenean silver fir forests. Forest Ecology and Management 262:759-769.

Camarero, J.J., Sangüesa Barreda, G., Alla, A.Q., González de Andrés, E., Maestro Martínez, M., Vicente-Serrano, S.M. 2012. Los precedentes y las respuestas de los árboles a sequías extremas revelan los procesos involucrados en el decaimiento de bosques mediterráneos de coníferas. Ecosistemas 21(3):22-30.

Candel-Pérez, D., Lucas-Borja, M.E., Linares, JC. 2012. Predicciones del crecimiento en poblaciones de pino laricio (Pinus nigra Arn. ssp. salzmanii) bajo diferentes escenarios futuros de cambio climático. Ecosistemas 21(3):41-49.

Carnicer, J., Coll, M., Ninyerola, M., Pons, X., Sanchez, G., Penuelas, J. 2011. Widespread crown condition decline, food web disruption, and amplified tree mortality with increased climate change-type drought. Proceedings of the National Academy of Science USA. 108:1474-1478.

Cook, E.R. 1985. A time series analysis approach to tree-ring standardization. PhD Thesis, University of Arizona, Tucson.

Dobbertin, M. 2005. Tree growth as indicator of tree vitality and of tree reaction to environmental stress: a review. European Journal of Forest Research. 124:319-333

Drobyshev, I., Linderson, H., Sonesson, K. 2007. Relationship Between Crown Condition and Tree Diameter Growth in Southern Swedish Oaks. Environmental Monitoring Assessment 128:61-73.

Duchesne, L., Ouimet, R., Morneau, C. 2003. Assessment of sugar maple health based on basal area growth pattern. Canadian Journal of Forest Research 33(11):2074-2080.

Eid, T., Tuhus, E. 2001. Models for individual tree mortality in Norway. Forest Ecology and Management 154:69-84.

Fernández-Cancio, A., Navarro Cerrillo, R.M., Sánchez-Salguero, R., Fernández Fernández, R., Manrique Menéndez, E. 2011. Viabilidad fitoclimática de las repoblaciones de pino silvestre (Pinus sylvestris L.) en la Sierra de los Filabres (Almería). Ecosistemas 20(1):124-144.

Fritts, H.C. 1976. Tree Rings and Climate. Academic Press, New York. USA.

Galiano, L., Martínez-Vilalta, J., Lloret, F. 2010. Drought-Induced Multifactor Decline of Scots Pine in the Pyrenees and Potential Vegetation Change by the Expansion of Co-occurring Oak Species. Ecosystems 13:978-991. 
Galiano, L., Martínez-Vilalta, J., Lloret, F. 2011. Carbon reserves and canopy defoliation determine the recovery of Scots pine 4 year after a drought episode. New Phytologist 190 (3):750-759.

Gómez-Aparicio, L., García-Valdes, R., Ruiz-Benito, P., Zavala, M.A. 2011 Disentangling the relative importance of climate, size and competition on tree growth in Iberian forests: implications for management under global change. Global Change Biology 17:2400-2414.

Gutiérrez, E. 1989. Dendroclimatological study of Pinus sylvestris L. in southern Catalonia (Spain). Tree-Ring Bulletin 49:1-9.

Helama, S, Salminen, H, Timonen, M, Varmola, M. 2008. Dendroclimatological analysis of seeded and thinned Scots pine (Pinus sylvestris L.) stands at the coniferous timberline. New Forests 35:267-284.

Hernández-Clemente, R., Navarro-Cerrillo, R.M., Suarez, L., Morales, F, Zarco-Tejada, P.J. 2011. Assessing structural effects on PRI for stress detection in conifer forests. Remote Sensing and Environment. 115:2360-2375.

Holmes, R.L. 1983. Computer-assisted quality control in tree-ring dating and measurement. Tree-Ring Bulletin 43:68-78.

IPCC 2007. Climate Change 2007: The Physical Science Basis. Cambridge, UK. Cambridge University Press, pp 996.

Juknys, R., Vencloviene, J., Stravinskiene, V., Augustaitis, A., Bartkevicius, E. 2003. Scots pine (Pinus sylvestris L.) growth and condition in a polluted environment: from decline to recovery. Environmental Pollution 125:205-212.

Jump, A.S., Hunt, J.M., Peñuelas, J., 2006. Rapid climate change-related growth decline at the southern range edge of Fagus sylvatica. Global Change Biology 12:2163-2174.

Linares, J.C., Camarero, J.J., Carreira, J.A. 2009. Interacting effects of changes in climate and forest cover on mortality and growth of the southernmost European fir forests. Global Ecology and Biogeography 18:485-497.

Linares, J.C., Camarero, J.J., Carreira, J.A. 2010. Competition modulates the adaptation capacity of forests to climatic stress: insights from recent growth decline and death in relict stands of the Mediterranean fir Abies pinsapo. Journal of Ecology 98:592-603.

Linares, J.C., Pazo Sarria, R., Taïqui, L., Camarero, J.J., Ochoa, V., Lechuga, V., Seco, J.I., Viñegla, B., Sangüesa, G., Gilarte, P., Merino, J. 2012. Efectos de las tendencias climáticas y la degradación del hábitat sobre el decaimiento de los cedrales (Cedrus atlantica) del norte de Marruecos. Ecosistemas 21(3):7-14.

Linares, J.C., Tíscar, PA. 2010. Climate change impacts and vulnerability of the southern populations of Pinus nigra subsp. salzmannii. Tree Physiology 30(7):795-806.

Lines, E.R., Coomes, D.A., Purves, D.W. 2010. Influences of forest structure, climate and species composition on tree mortality across the Eastern US. PLoS ONE 5:e13212.

Lloret, F. 2012. Vulnerabilidad y resiliencia de los ecosistemas terrestres frente a episodios extremos de sequía. Ecosistemas 21(3):85-90.

Lloret, F., Siscart, D. 1995. Los efectos demográficos de la sequía en poblaciones de encina. Cuadernos de la Sociedad Española de Ciencias Forestales 2:77-81.

LUCDEME 2004. Ministerio de Medio Ambiente, Proyecto LUCDEME (Provincia de Almería-Baza) 1986-2004. Memorias y mapas de suelos E. 1:50.000. Madrid, Spain.(http://www.mma.es/portal/secciones/biodiversidad/desertificacion/lucha_desertificacion/lucdeme.htm)

Macias, M., Andreu, L., Bosch, O., Camarero, J.J., Gutiérrez E. 2006. Increasing aridity is enhancing silver fir (Abies alba Mill.) water stress in its south-western distribution limit. Climatic Change 79:289-313.

Manion, P.D. 1981. Tree disease concepts. Upper Saddle River. Prentice Hall, NKJ, USA. 09 pp.

Manrique Menéndez, E., Fernández-Cancio, A. 2000. Extreme climatic events in dendroclimatic reconstructions from Spain. Climatic Change 44:123-138.

Martínez-Vilalta, J., Piñol, J. 2002. Drought-induced mortality and hydraulic architecture in pine populations of the NE Iberian Peninsula. Forest Ecology and Management 161:247-256.
Martínez-Vilalta, J., Sala, A., Piñol, J. 2004. The hydraulic architecture of Pinaceae. Plant Ecology 171:3-13.

Martínez-Vilalta, J., López, B.C., Adell, N., Badiella, L., Ninyerola, M. 2008. Twentieth century increase of Scots pine radial growth in NE Spain shows strong climate interactions. Global Change Biology 14:28682881.

Martínez-Vilalta, J., Aguadé, D., Banqué, M., Barba, J., Curiel Yuste, J., Galiano, L., Garcia, N., Gómez, M., Heres, A.M., López, B.C., Lloret, F., Poyatos, R. Retana, J. Sus, O., Vayreda, J., Vilà-Cabrera, A. 2012. Las poblaciones ibéricas de pino albar ante el cambio climático: con la muerte en los talones. Ecosistemas 21(3):15-2.

Millar, C.I., Stephenson, N.L., Stephens, S.L., 2007. Climate change and forests of the future: managing in the face of uncertainty. Ecological $A p$ plications 17:2145-2151.

Monserud, R.A., Sterba, H. 1996. A basal area increment model for individual trees growing in even- and uneven-aged forest stands in Austria. Forest Ecology and Management 80:57-80.

Mueller, E. Stierlin, H.R. (Ed.) 1990. Sanasilva tree crown photos with percentages of foliage loss, Birmensdorf, Switzerland.

Navarro-Cerrillo, R.M, Varo, M.A., Lanjeri, S., Hernández Clemente, R. 2007. Cartografía de defoliación en los pinares de pino silvestre (Pinus sylvestris L.) y pino salgareño (Pinus nigra Arn.) en la Sierra de los Filabres. Ecosistemas 16,163-171.

Palahí, M., Grau, J.M. 2003. Preliminary site index model and individual tree-growth and mortality models for black pine (Pinus nigra Arn.) in Catalonia (Spain). Investigación Agraria, Sistemas y Recursos Forestales 12(1):137-148

Peñuelas, J., Lloret, F., Montoya, R. 2001. Severe drought effects on Mediterranean woody flora. Forest Science. 47:214-218.

Pemán García, J., Navarro-Cerrillo, R.M. 1997. Repoblaciones Forestales. Universidad de Lérida. Universidad de Córdoba. Lérida. 378 pp.

Rigling, A., Brühlhart, H., Bräker, O.U.,Orster, T., Schweingruber, FH. 2003. Irrigation effect on tree growth and vertical resin duct production of Pinus sylvestris L. on dry sites in the Central Alps, Switzerland. Forest Ecology and Management 163:105-121.

Sánchez-Salguero, R. 2012. Forest decline in pine plantations under climate change at southern Iberian Peninsula. Dendroecology and modeling. $\mathrm{PhD}$ Thesis, University of Córdoba, Córdoba, Spain. Disponible en: http://dl.dropbox.com/u/15696914/Sanchez-Salguero.Rdissertation May2012.pdf

Sánchez-Salguero, R., Navarro-Cerrillo, R.M., Camarero, J.J., FernándezCancio, A. 2010. Drought-induced growth decline of Aleppo and maritime pine forests in south-eastern Spain. Forest Systems 19:458-469.

Sánchez-Salguero, R., Navarro-Cerrillo RM., Camarero J.J., FernándezCancio A. 2012a. Selective drought-induced decline of pine species in southeastern Spain. Climatic Change 113:767-785.

Sánchez-Salguero, R., Navarro-Cerrillo, R.M., Swetnam, T.W., Zavala, M.A. $2012 \mathrm{~b}$. Is drought the main decline factor at the rear edge of Europe? The case of southern Iberian pine plantations. Forest Ecology and Management 271:158-169.

Serrada Hierro, R., Montero González, G., Reque, J. (Eds). 2008. Compendio de Selvicultura Aplicada en España. INIA-Fundación Conde del Valle de Salazar. Madrid, Spain. 1178 pp.

Tardif, J., Camarero, J.J. Ribas, M., Gutiérrez, E. 2003. Spatiotemporal variability in tree ring growth in the Central Pyrenees: climatic and site influences. Ecological Monographs, 73:241-257.

Vilá-Cabrera, A., Martínez-Vilalta, J., Vayreda, J., Retana, J. 2011. Structural and climatic determinants of demographic rates of Scots pine forests across the Iberian Peninsula. Ecological Applications 21(4):1662-1172.

Wigley T.M.L, Briffa K.R, Jones P.D. 1984. On the average value of correlated time series, with applications in dendroclimatology and hydrometeorology. Journal of Applied Meteorology and Climatology 644 23:201-213.

Yamagughi, D.K. 1991. A simple method for cross-dating increment cores from living trees. Canadian Journal of Forest Research. 21:414-416. 